\title{
COVID-19-associated pneumomediastinum
}

\author{
Authors: Nina Goldman, ${ }^{\mathrm{A}}$ Balamayran Ketheeswaran ${ }^{\mathrm{B}}$ and Henrietta Wilson ${ }^{\mathrm{C}}$
}

A case is presented highlighting the emerging association of COVID-19 with pneumomediastinum, even in patients who have never received mechanical ventilation or positive airway pressure.

KEYWORDS: COVID-19, SARS, pneumomediastinum

DOI: 10.7861/clinmed.2020-0247

\section{Case presentation}

A 64-year-old man with a background of type 2 diabetes and obstructive sleep apnoea (OSA) treated with home continuous positive airway pressure (CPAP) presented to hospital with new pleuritic chest pain and tachycardia.

He had been discharged 5 days previously following a 3-week admission to the same hospital with laboratory-confirmed COVID-19. During his previous admission he had required simple oxygen support only, with a maximum oxygen requirement of $4 \mathrm{~L}$ via nasal cannula. He did not use his home CPAP and never required mechanical ventilation during admission. He had been discharged with saturations of $94 \%$ on room air and reported no use of his home CPAP following discharge.

On admission, arterial blood gas demonstrated $\mathrm{aPaO}_{2}$ of $12 \mathrm{kPa}$ on room air. Blood tests including full blood count and C-reactive protein were within normal range.

A repeat chest $X$-ray showed ongoing ground-glass opacification of both lungs similar to on previous discharge (Fig 1). He went on to have a computed tomography (CT) pulmonary angiography which did not reveal a pulmonary embolism but showed pneumomediastinum as well as ongoing consolidation consistent with COVID-19. No pneumothorax and no evidence of oesophageal perforation was seen on CT (Fig 2).

Following admission, the patient remained stable with no additional treatment required. He was therefore discharged after 24 hours observation. A 6-week outpatient follow-up with repeat chest $\mathrm{X}$-ray was arranged.

\section{Discussion}

Pneumomediastinum is a rare condition in which air is present in the mediastinum. It more commonly affects males and frequently

Authors: Arespiratory registrar, Whipps Cross University Hospital, London, UK; Brespiratory senior house officer, Whipps Cross University Hospital, London, UK; ' Cardiothoracic surgeon, St Bartholomew's Hospital, London, UK

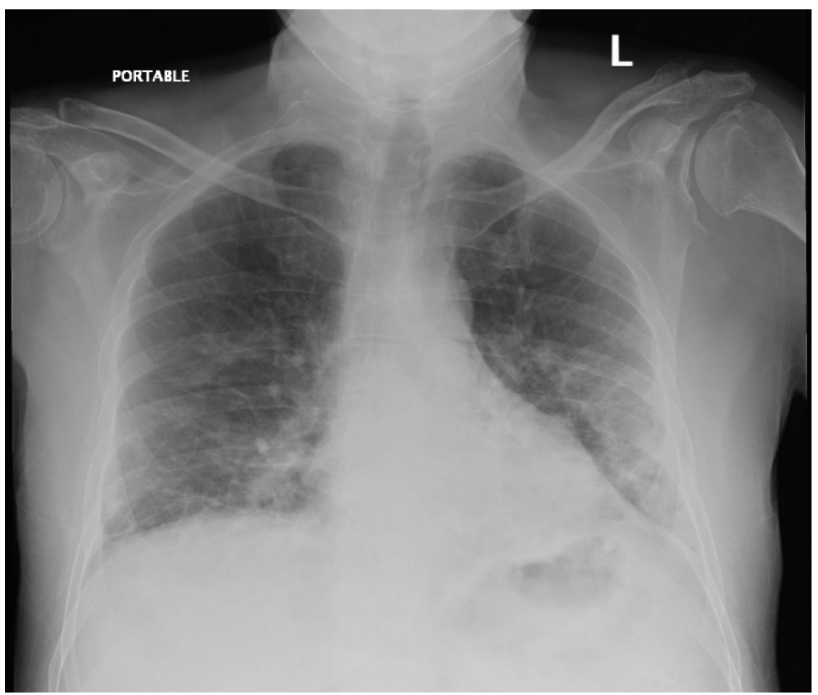

Fig 1. Chest X-ray showing bilateral ground glass opacification and bands of linear atelectasis in right lung base.

occurs as a result of blunt trauma or oesophageal perforation. It is also a well-known complication of barotrauma of the chest including positive airway pressure and mechanical ventilation. Other associated risk factors include smoking and pre-existing lung parenchymal and airways disease.'

Most commonly patients present with shortness of breath, cough and/or chest or neck pain. Physical signs can include tachycardia, tachypnoea, hypotension and subcutaneous emphysema. Pneumomediastinum may be visible on plain chest X-ray; however, if small it may only be detected on CT of the chest. Spontaneous pneumomediastinum is usually self-limiting with no interventions required. Rarely, in cases of tension pneumomediastinum, severe cardio-pulmonary compromise can occur.

Pneumomediastinum unrelated to mechanical ventilation has been reported in COVID-19 and severe acute respiratory syndrome (SARS). ${ }^{2,3}$ In SARS, mean presentation of pneumomediastinum was 19.6 days post symptom onset. ${ }^{3}$ Pneumomediastinum may result from diffuse alveolar damage leading to alveolar rupture and interstitial emphysema with air dissecting along the bronchovascular sheaths into the mediastinum. ${ }^{3}$

Several treatments for COVID-19 are being used as part of clinical trials, off-label and on a compassionate-use basis. However currently no treatments have been approved as safe and effective. ${ }^{4}$ The search for COVID-19 treatments to reduce 

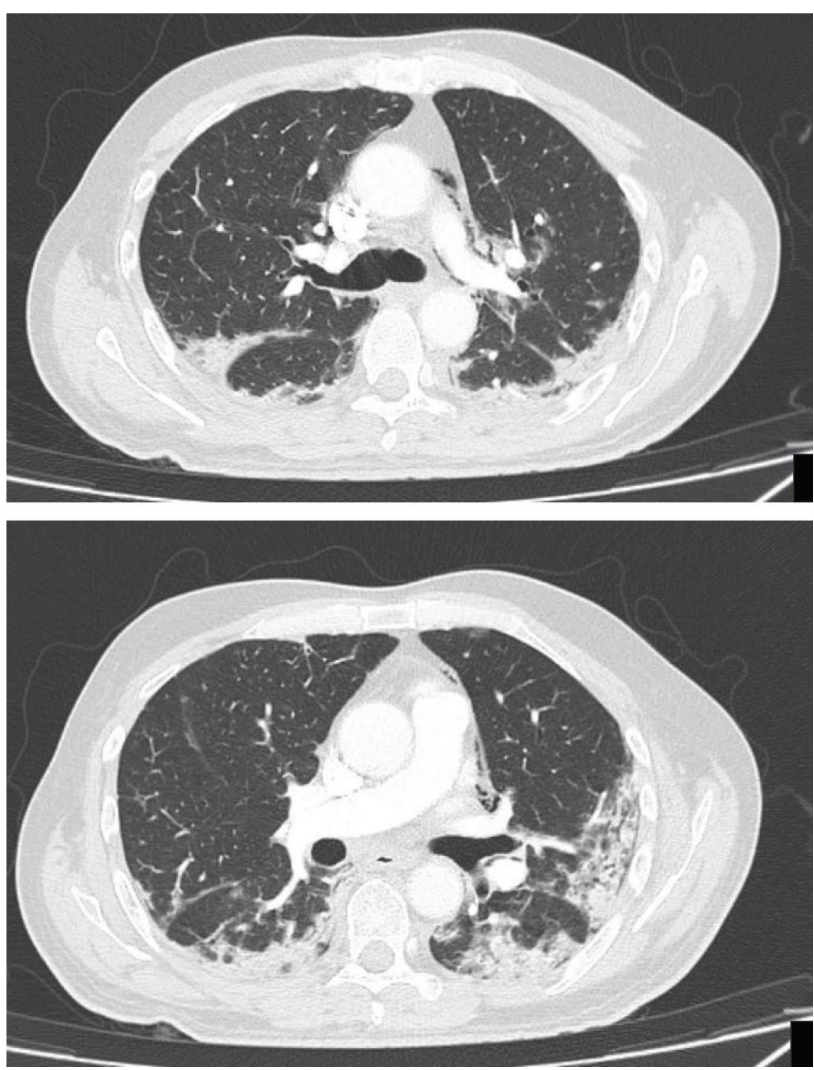

Fig 2. Multiple axial slices of a computed tomography pulmonary angiography. Multifocal peripheral textured ground glass opacification and consolidation consistent with COVID-19 seen. In addition, significant pneumomediastinum seen, with multiple locules of gas noted in the anterior mediastinum and along the main left pulmonary artery. transmission, improve outcomes and reduce complications such as pneumomediastinum continues.

This case highlights the emerging association of COVID-19 with pneumomediastinum and the need to be alert to this complication even in patients who never received mechanical ventilation or positive airway pressure support. Further research is needed into the exact pathogenesis and prevalence of this complication.

\section{References}

1 Caceres M, Ali SZ, Braud R, Weiman D, Garrett HE. Spontaneous pneumomediastinum: a comparative study and review of the literature. Ann Thorac Surg 2008;86:962-6.

2 Zhou C, Gao C, Xie Y, Xu Ml. COVID-19 with spontaneous pneumomediastinum. Vol. 20, Lancet Infect Dis 2020;20:510.

3 Chu CM, Leung YY, Hui JYH et al. Spontaneous pneumomediastinum in patients with severe acute respiratory syndrome. Eur Respir ] 2004;23:802-4.

4 World Health Organization. Clinical management of severe acute respiratory infection (SARI) when COVID-19 disease is suspected. WHO, 2020. Available from www.who.int/publications-detail/ clinical-management-of-severe-acute-respiratory-infection-whennovel-coronavirus-(ncov)-infection-is-suspected [Accessed 10 May 2020].

Address for correspondence: Dr Nina Goldman, Whipps Cross Hospital, Whipps Cross Road, London E11 1NR, UK. Email: nina.goldman@nhs.net 